\title{
Correction to: Phosphomimetic Mutation of Glycine Transporter GlyT1 C-Terminal PDZ Binding Motif Inhibits its Interactions with PSD95
}

\author{
Martina Baliova $^{1} \cdot$ Frantisek Jursky $^{1}$ (D) \\ Published online: 9 January 2020 \\ (C) Springer Science+Business Media, LLC, part of Springer Nature 2020
}

Correction to: Journal of Molecular Neuroscience

https://doi.org/10.1007/s12031-019-01435-4

The original version of this article unfortunately contained two mistakes. In the html version, Fig. 1 was missing and was replaced by Fig. 2, resulting to identical version of both figures. While in the PDF version, Figs. 1 and 2 were inadvertently interchanged.

The correct figures are presented in the next page.

The original article has been corrected.

The online version of the original article can be found at https://doi.org/ 10.1007/s12031-019-01435-4

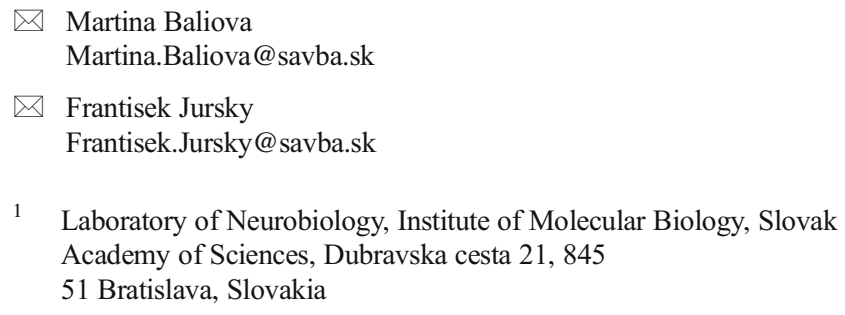

1 Laboratory of Neurobiology, Institute of Molecular Biology, Slovak Academy of Sciences, Dubravska cesta 21, 845

51 Bratislava, Slovakia 


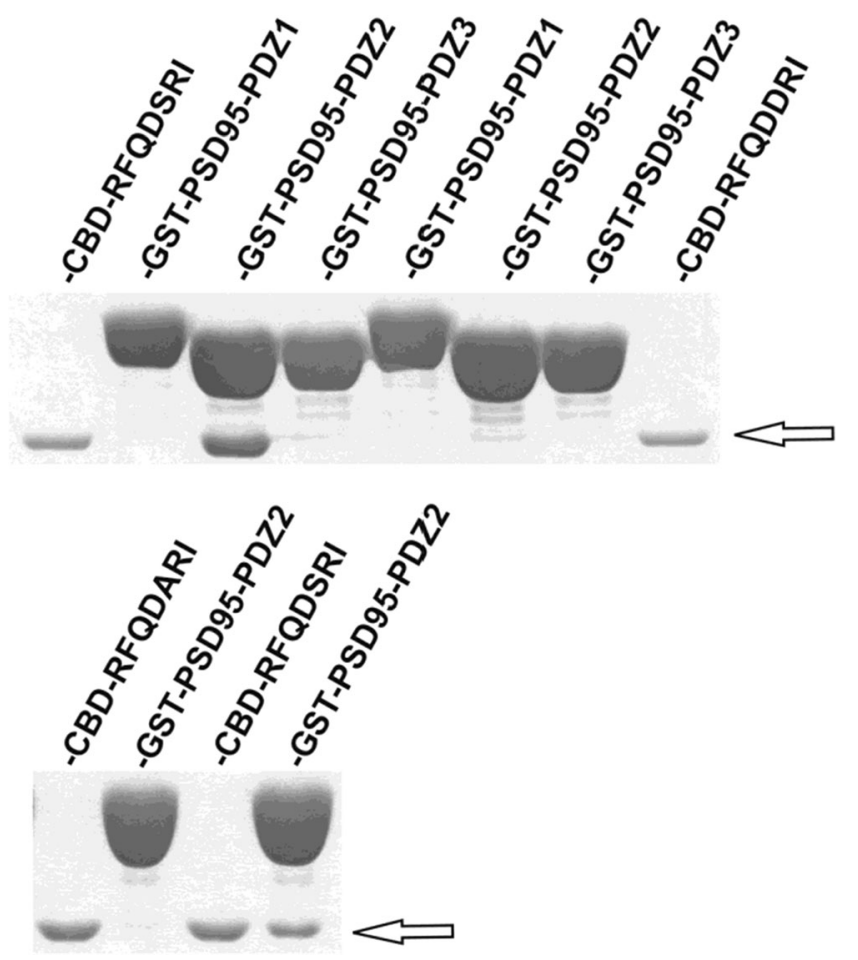

Fig. 1 Interaction of the CBD-RFQDSRI fusion protein its phosphomimetic CBD-RFQDDRI and neutral variant CBD-RFQDARI (marked by arrow) with GST fusion proteins containing PDZ domains 1, 2 , and 3 of the PSD95 protein. The PDZ motifs fused with small cellulose-binding proteins TAG (CBD) were overexpressed in E. coli, sedimented cells were sonicated, and crude supernatants after centrifugation were interacted for $1 \mathrm{~h}$ at room temperature with GSTPSD95-PDZ domains 1,2 , and 3 proteins immobilized onGlutathione Sepharose resin. Following washing and elution, samples were resolved in $12 \%$ polyacrylamide gel and stained with Coomassie dye. Simulation of phosphorylation on GlyT1b serine 649 in the -SRI PDZ binding motif with -DRI sequence completely prevented the interaction with PDZ domain 2. The absence of interaction in the neutral -ARI mutant indicates high stringency of the sequence requirement for this position in the PDZ motif

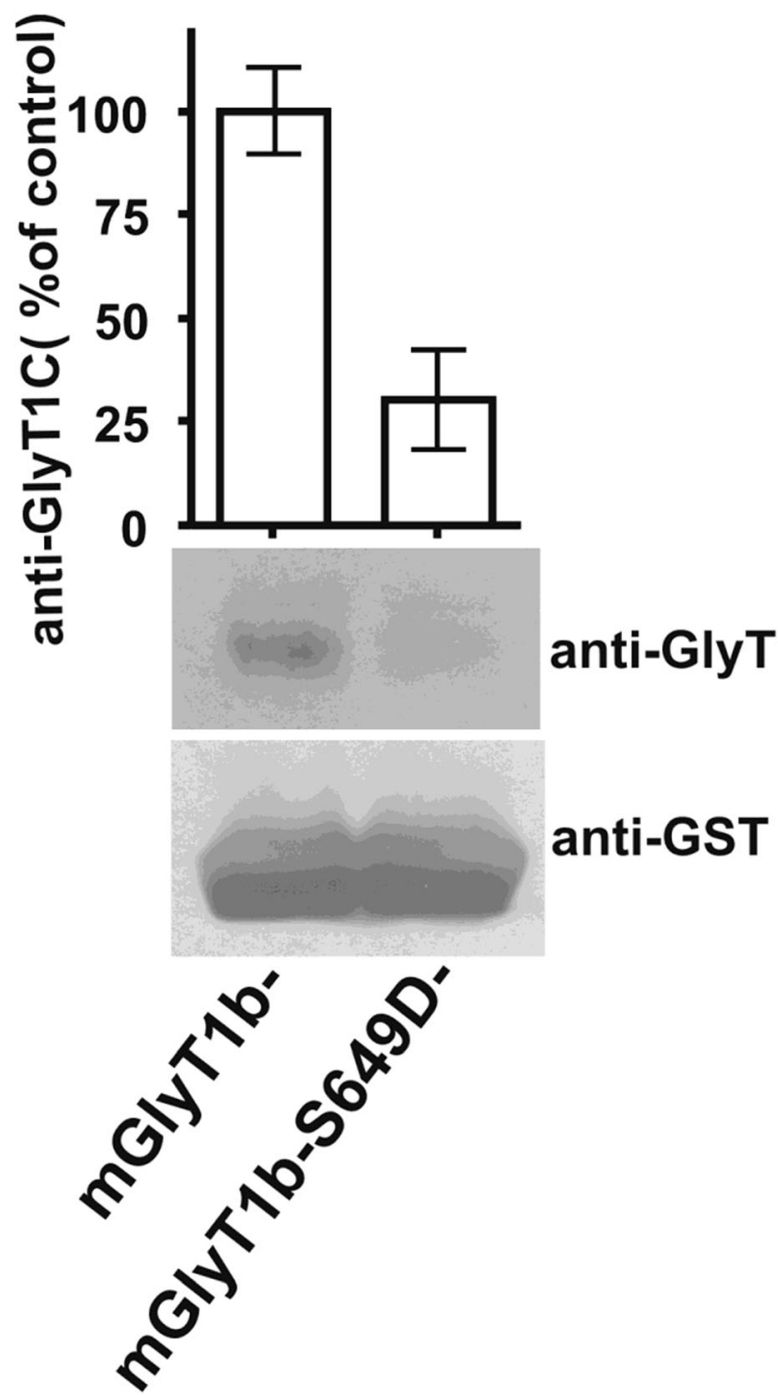

Fig. 2 Phosphomimetic inhibition of GlyT1 PDZ interaction with PSD95 PDZ domain 2. Wild type mGlyT1b transporter and its S649D mutant were transiently expressed in the N2a cell line. Cells were washed and solubilized with interaction buffer containing $1 \%$ Triton-X100, and the extract was interacted with GST-PSD95-PDZ2 fusion protein containing resin. Following washing and elution, samples were resolved in $7.5 \%$ polyacrylamide gel and immunoblotted with anti-GlyT1C and anti-GST antibodies. Graph data represent the mean ( \pm standard deviation, SD) of three independent experiments

Publisher's Note Springer Nature remains neutral with regard to jurisdictional claims in published maps and institutional affiliations. 